\title{
Synthesis of acrylic acid and its esters with Se-containing catalysts
}

\author{
Tetiana Kharandiuk ${ }^{1}$, Kok Hui Tan ${ }^{2}$, Volodymyr Ivasiv ${ }^{1}$, Roman Nebesnyi $^{1}$, Andrij Pich ${ }^{2}$ \\ 1. Department of Organic Products Technology, Lviv Polytechnic National University, UKRAINE, Lviv, 12 Stepan \\ Bandera st., E-mail: roman.v.nebesnyi@lpnu.ua
}

2. DWI Leibniz Institute for Interactive Materials e.V, GERMANY, Aachen, Forckenbeckstraße 50, E-mail: tan@dwi.rwth-aachen.de

\begin{abstract}
Se-containing catalysts of organic and inorganic nature were explored for the synthesis of acrylic acid and its esters in different solvents. Newly synthesized Se-containing microgel catalyst shown the best catalytic performance. With microgel catalyst different acrylate monomers can be produced with high yield simply by varying solvent nature.
\end{abstract}

Keywords - acrolein, acrylic acid, methyl acrylate, ethyl acrylate, butyl acrylate, Se-microgel catalysts.

\section{Introduction}

Acrylic acid and acrylate esters are widely used in industrial purposes for textile, adsorbent polymer, paints, adhesives manufacture [1]. Main method to produce acrylic acid is 2 step synthesis, that include propylene oxidation to acrolein with further acrolein oxidation to acrylic acid [2]. Acrylic acid can be used without further treatment or esterified to acrylate esters. Another method to produce acrylate esters is one-step oxidative esterification of acrolein [3]. Previously reported results $[3,4]$ shown, that up to $85 \%$ of methyl acrylate yield can be reached by this method. But to achieve such result, either reaction temperature has to be high $\left(250{ }^{\circ} \mathrm{C}\right)$ and catalyst, that contain hazardous heavy metals, has to be used [4] or reaction can be performed at moderate temperature $\left(25^{\circ} \mathrm{C}\right)$ but reaction has to last $40 \mathrm{~h} \mathrm{[3]}$.

To improve process of acrolein oxidation to acrylic acid or oxidative esterification to acrylate esters, reaction should be performed under mild conditions, during reasonable time, with minimum wastes and with high yield of product. Selenium catalysts shown to be effective in oxidation processes [5], and together with hydrogen peroxide as oxidant can provide good results under mild and green conditions [6]. For this purposes, we studied process of acrolein oxidation with hydrogen peroxide and selenium catalysts of organic and inorganic nature.

\section{Results and discussion}

In term to study the effect of catalyst nature on acrolein oxidation and oxidative esterification, reactions were performed at $50{ }^{\circ} \mathrm{C}$ with catalysts $0.0044 \mathrm{mmol} / \mathrm{ml}$ concentration, reaction time $-8 \mathrm{~h}$, oxidant - hydrogen peroxide. Methanol, ethanol, butanol and dioxane were used as solvents. $\mathrm{H}_{2} \mathrm{SeO}_{3}$, diphenyl diselenide (DPDS), diselenide crosslinker (X-linker, Fig. 1a), Se-modified microgel (Fig. 1b) were used as Se-containing catalysts.

Se-modified microgel consists of diselenide crosslinker, incorporated into $\operatorname{poly}(N$ vinylcaprolactam) microgels together with a permanent $N, N^{\prime}$-methylenebis(acrylamide) crosslinker. Microgel, as polymer colloid with open porous structure, surface activity and solvent resistance, can provide unique chemical functionality and catalytic activity of modified microgel.

Catalytic performance of Se-containing catalysts was evaluated by the yields of methyl acrylate and acrylic acid for the reaction performed in methanol (Fig. 2). All tested catalysts proved to be catalytically active in oxidative esterification of acrolein and allowed to reach up to $90 \%$ total yield of reaction. Regardless the catalyst, methyl acrylate yield was much higher than acrylic acid. This results are in good correlation with results in work [3], where authors explain higher yield of ester due to easier formation of intermediate compound in ester synthesis than in 
carboxylic acid synthesis. Diphenyl diselenide, X-linker and $\mathrm{H}_{2} \mathrm{SeO}_{3}$ shown similar results. Semodified microgel shown the highest yield of reaction $-89 \%$.

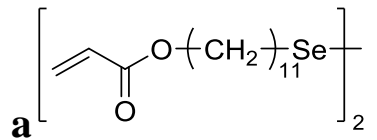

b

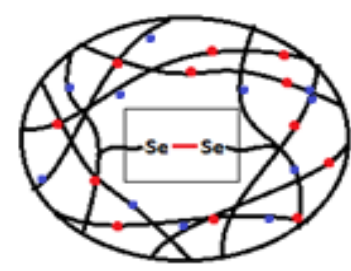

Fig. 1. a - Diselenide crosslinker; $\mathrm{b}-$ Se-modified microgel

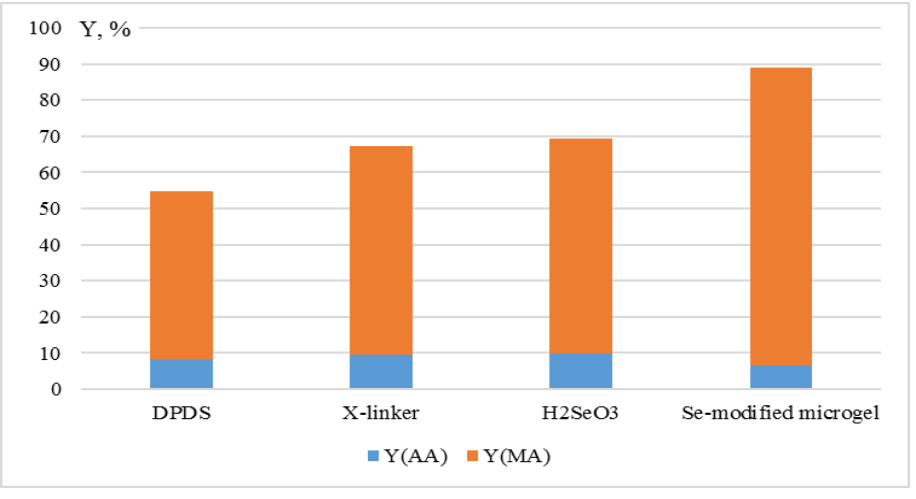

Fig.2. Effect of catalyst nature on acrylic acid (AA) and methyl acrylate (MA) yield

To study the effect of solvent nature on the synthesis of acrylic acid and acrylate esters, Semodified microgel was used as the most effective catalyst. In methanol medium, ester yield and ester:acid ratio was the highest $-83 \%$ and 12:1 respectively. With increase of hydrocarbon chain in alcohol molecule $\left(\mathrm{C}_{2} \mathrm{H}_{5}-\mathrm{OH}\right.$ and $\left.\mathrm{C}_{4} \mathrm{H}_{9}-\mathrm{OH}\right)$ ester:acid ratio decreases, but ester yield is still higher than acid. In dioxane medium only acrylic acid was formed with $79 \%$ yield.

\section{Conclusion}

Acrylic acid and acrylate esters can be easily synthesised in one step reaction from acrolein with yield up to $79 \%$ and $83 \%$ respectively with Se-modified microgel catalyst. Products of the reaction and their ratio can me modified by chosen solvent.

\section{Acknowledgments}

The authors acknowledge Volkswagen Stiftung for the project A115859 funding.

\section{References}

[1] R. Beerthuis, G. Rothenberg, N. R. Shiju, "Catalytic routes towards acrylic acid, adipic acid and $\varepsilon$-caprolactam starting from biorenewables," Green Chemistry, vol. 17, no. 3, March, pp. 1341-1361, 2015.

[2] K. Weissermel and H.-J. Arpe, Industrial Organic Chemistry. Weinheim: WILEY-VCH Verlag GmbH \& Co. KGaA, 2003.

[3] C. Marsden, E. Taarning, D. Hansen, L. Johansen, S. K. Klitgaard, K. Egeblad and C. H. Christensen, "Aerobic oxidation of aldehydes under ambient conditions using supported gold nanoparticle catalysts," Green Chemistry, vol. 10, no. 2, Feb., pp. 168-170, 2008.

[4] K. Yu. Koltunov, V. I. Sobolev, V. M. Bondareva, "Oxidation, oxidative esterification and ammoxidation of acrolein overmetal oxides: Do these reactions include nucleophilic acyl substitution?," Catalysis Today, vol. 279, no. 1, Jan., pp. 90-94, 2017.

[5] L. Zhou, B. Donga, S. Tang, H. Ma, C. Chen, X. Yang, J. Xu, "Sulfonated carbon catalyzed oxidation of aldehydes to carboxylic acids by hydrogen peroxide," Journal of Energy Chemistry, vol. 22, no. 4, Jul., pp. 659-664, 2013.

[6] Y. Rangraz, F. Nemati, A. Elhampour, "Diphenyl diselenide immobilized on magnetic nanoparticles: A noveland retrievable heterogeneous catalyst in the oxidation of aldehydes under mild and green conditions," Journal of Colloid and Interface Science, vol. 509, no. 1, Jan., pp. 485-494, 2018. 